\title{
Reactive Real-Time Cooperation as a Combination of Altruism and Self Satisfaction
}

\author{
Olivier Simonin and Jacques Ferber \\ LIRMM - Université Montpellier II, \\ 161 rue Ada, 34392 Montpellier Cedex 5, France \\ \{simonin,ferber\}@lirmm.fr
}

\section{Introduction}

This work proposes a method for integrating cooperative behaviors among distributed autonomous agents based on local communications. The presented architecture is reactive-based to ensure self-organization, reactivity and adaptability of the agents.

Animal signal spreading have interesting characteristics such as spatial limited communication, intensity meaning, redundant information (robustness) and reactivity. We investigate this type of communication to improve reactive architecture in order to handle robust communication and reactive cooperation.

The underlying idea of our architecture is to use a signal of interactive satisfaction to perform action selection and cooperation. By taking into account the satisfaction of its acquaintances, an agent can have an altruistic behavior thus allowing for intentional cooperation between agents.

\section{Agent Satisfaction}

From the designer point of view, the agent behavior must satisfy goal-oriented, conservative and cooperative functions. The principle of our agent model relies on the maximization of agent satisfactions. At any time, the agent try to maximize either its selfish interests or the collective interests.

As motions are involved in a majority of situated agent tasks, we investigate in priority spatial cooperation and spatial conflicts resolution. We define the agent behavior as a combination of selected goals which can either be individual goals or altruistic responses to requests for cooperation.

In our model, agent satisfaction is composed of three different sub-satisfactions: personal satisfaction, interactive satisfaction and empathy satisfaction.

For an agent, the first two sub-satisfactions express the progress of its individual goal (= self satisfaction). The personal satisfaction $P$ measures only the progress of the agent's task. The interactive satisfaction $I$ is a value computed as a reaction or an intention to agent acquaintances actions. It expresses the quality of interaction between the agent and its neighbors. Lastly, the empathy satisfaction $E$ is the average value of personal satisfaction of its acquaintances, it expresses the altruism of the agent.

\section{Modeling agent altruism}

Agents broadcast only their interactive satisfaction $I$ because it expresses the reaction to other agents behaviors. This signal is broadcasted within a bounded distance from the agent. The intensity and the variation of the signal define the meaning of the communication. We note $I_{i}(t)$ the level of interactive satisfaction emitted by the agent $i$ at time $t$. Let $\Delta I_{i}(t)_{T}$ be the function of the interactive satisfaction variation, $\Delta I_{i}(t)_{T}=I_{i}(t)-I_{i}(t-T)$. It represents the satisfaction evolution of an agent relative to its acquaintance actions between times $t-T$ and $t$.

In the vector model, the cooperative behavior is defined with the altruism vector $\vec{\vartheta}(t)$. Formally, the altruism vector of an agent (B) relative to another agent (A) $\bar{\vartheta}_{B / A}^{-}-\overrightarrow{(t)}$ is computed as follows:

$$
\begin{array}{r}
\bar{\vartheta}_{B_{/ A}}^{-}\left(\overrightarrow{t)}=k \cdot S\left(\Delta I_{A_{\text {A }}}\right) \cdot \frac{\left|I_{A_{\text {AB }}}(t)\right|}{\operatorname{dist}_{A, B}^{2}} \cdot \vec{B} \vec{A}\right. \\
S(x(t))= \begin{cases}\operatorname{Sign}(x(t)) & \text { if } x(t) \neq 0 \\
\operatorname{Sign}(x(t-T)) & \text { if } x(t)=0\end{cases}
\end{array}
$$

Equation 1 is like a force deriving from a repulsive field. Our approach consist to transform the signal of interactive satisfaction into a mathematical vector directly added to the agent motion vector. Because of the reactive communication, agents move in real time into dynamical vector fields.

As $\Delta I_{A_{/ B}}$ may be null, we have defined a sign function $S$ to avoid a null vector $\bar{\vartheta}_{B_{/ A}}-\overrightarrow{(t)}$ and to ensure the continuation of a reaction. 


\section{Sequential Signal-Passing:}

- When agents are in a spatial deadlock situation, a chain process of dissatisfaction emission is performed until agents may move.

- When an agent follows a signal with a positive increase, it emits also an attractive signal in order to call its own acquaintances. The force of the signal variation must decline during the propagation in order to limit the number of attracted agents.

Multi-agent Altruism: A situated agent close to others can simultaneously perceive several attractions and repulsions. In order to define a new altruism vector we divide the multi-agent problem in a set of inter-agent problems. The altruistic reaction of agent $B$ close to $N$ acquaintances (noted $\Lambda$ ) is computed as follows

$$
\bar{\vartheta}_{B_{/ \Lambda}}^{-} \stackrel{\longrightarrow}{=} \sum_{j \in \Lambda} k \cdot S\left(\Delta I_{j_{/ B}}\right) \cdot \frac{\left|I_{j_{/ B}}(t)\right|}{d i s t_{j, B}^{2}} \cdot \bar{B} \vec{J}
$$

\section{Cooperative architecture}

Our architecture is built on a classical schema-based reactive control [1] using vector combination [3].

At any time, vegetative tasks may be released following internal and environmental stimulus. If no one is triggered, the architecture computes the new current goal as a selection between the current task, a new task and the altruism vector.

If the current task is a not finish altruistic behavior, this task is again selected. Otherwise, a new task may be selected if its stimulus force is greater than the current task performance index (= personal satisfaction $P$ ).

But, if the more powerful perceived signal $I$ is greater than intensity of the current task, the agent select the altruism vector derived from $I$ as current task.

Finally, if the the current task is a moving action, the computed vector is a linear function of the goal vector (which may be the altruism vector), the obstacles avoiding vector and the altruistic vectors of the perceived repulsive signals (with scalar weigts on vectors).

Figure 1 shows the principle of action selection/combination using the personal and interactive satisfaction.

\section{Experimental results}

A 2D simulation of a foraging-consuming problem have been implemented to test our model. Simulated robots are based on the satisfaction architecture and simple primitive behaviors (tasks). They have to move in a constrained environment, find and exploit mines, avoid agent hindrances and recharge their energy.

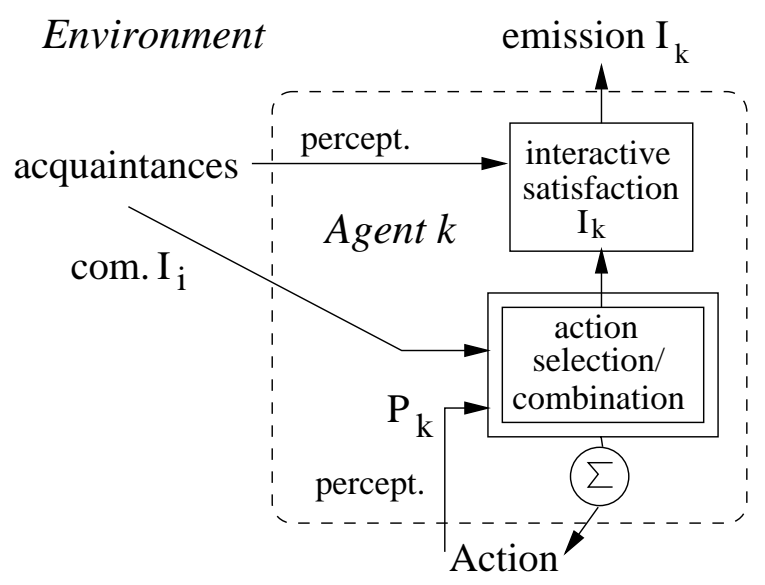

Figure 1. Principle of the architecture

In every simulation, robots efficiently explore the environment and slide around obstacles. They frequently compute an altruistic behavior to avoid mutual hindrances, and as a consequence they are homogeneously distributed.

Robots extract and transport back the raw material until mines are worked out. Moreover, they adapt their behavior to each dynamic event: moves of robots, working out of mines and need for energy. Details and quantitative results of these simulations can be found in [2].

\section{Conclusion}

The model abstracted here shows the feasibility of using agents intentions in a reactive architecture to solve the problem of action selection/combination. We have seen that reactive cooperation relies on simple signals and the propagation of satisfactions between agents. This model remains within the vector field approach and provides for real-time cooperation.

Finally, experimental results illustrate the ability of the model to achieve efficient navigation, adaptive and reactive cooperation in collective robotics application.

Future works will address the study of parameter variations effect on performances, the introduction of learning and its application to real mobile robots.

\section{References}

[1] R. Arkin. Motor schema based mobile robot navigation. International Journal of Robotics Research, pages 92-112, 1989.

[2] O. Simonin and J. Ferber. Modeling self satisfaction and altruism to handle action selection and reactive cooperation. In SAB'2000, to appear, Paris, France, 2000.

[3] K. Zeghal. A comparaison of different approaches based on force fields for coordination among multiple mobiles. In IEEE International Conference on Intelligent Robots and Systems (IROS), Victoria, B.C., Canada, October 13-17 1998. 